\title{
3D image-based adapted high-dose-rate brachytherapy in cervical cancer with and without interstitial needles: measurement of applicator shift between imaging and dose delivery
}

\author{
Leif Karlsson, PhLic',2, Per Thunberg, PhD², Anders With, MSc², Lovise Bohr Mordhorst, MD',3, Jan Persliden, PhD',2 \\ ISchool of Health and Medical Sciences, ${ }^{2}$ Department of Medical Physics, Faculty of Medicine and Health, ${ }^{3}$ Department of Oncology, Faculty \\ of Medicine and Health, Örebro University. Örebro, Sweden
}

\begin{abstract}
Purpose: Using 3D image-guided adaptive brachytherapy for cervical cancer treatment, it often means that patients are transported and moved during the treatment procedure. The purpose of this study was to determine the intra-fractional longitudinal applicator shift in relation to the high risk clinical target volume (HR-CTV) by comparing geometries at imaging and dose delivery for patients with and without needles.

Material and methods: Measurements were performed in 33 patients (71 fractions), where 25 fractions were without and 46 were with interstitial needles. Gold markers were placed in the lower part of the cervix as a surrogate for HR-CTV, enabling distance measurements between HR-CTV and the ring applicator. Shifts of the applicator relative to the markers were determined using planning computed tomography (CT) images used for planning, and the radiographs obtained at dose delivery. Differences in the physical $D_{90}$ for HR-CTV due to applicator shifts were simulated individually in the treatment planning system to provide the relative dose variation.

Results: The maximum distances of the applicator shifts, in relation to the markers, were $3.6 \mathrm{~mm}$ (caudal), and $-2.5 \mathrm{~mm}$ (cranial). There was a significant displacement of $-0.7 \mathrm{~mm}(\mathrm{SD}=0.9 \mathrm{~mm})$ without needles, while with needles there was no significant shift. The relative dose variation showed a significant increase in $\mathrm{D}_{90} \mathrm{HR}-\mathrm{CTV}$ of $1.6 \%$ $(\mathrm{SD}=2.6 \%)$ when not using needles, and no significant dose variation was found when using needles.

Conclusions: The results from this study showed that there was a small longitudinal displacement of the ring applicator and a significant difference in displacement between using interstitial needles or not.

J Contemp Brachytherapy 2017; 9, 1: 52-58 DOI: https://doi.org/10.5114/jcb.2017.66110
\end{abstract}

Key words: brachytherapy, cervical cancer, intra-fraction, HDR.

\section{Purpose}

Modern treatment of advanced cervical cancer is a combination of external radiotherapy, concomitant chemotherapy, and brachytherapy $[1,2,3]$. High-dose-rate (HDR) or pulsed-dose-rate (PDR) brachytherapy plays a major role in the treatment. The steep dose gradient makes it possible to provide a very high central dose to the target while sparing the surrounding organs at risk (OAR), such as the bladder, rectum, and sigmoid. Localization and contouring of the target and OAR is needed to perform the evaluation of dose distribution.

The application of brachytherapy is specified in the European recommendations from the GEC-ESTRO (Groupe Européen de Curiethérapie European Society for Radiotherapy and Oncology) Working Groups $[4,5,6,7]$. These recommendations describe the $3 \mathrm{D}$ image-guided adaptive brachytherapy (IGABT) technique. One difference compared to the previously used technique is the use of magnetic resonance imaging (MRI) and/or computed tomography (CT) for outlining target and organ structures. These images are then also used for applicator reconstruction and dose optimization, which enables a final evaluation of dose distribution with dose volume histograms. Another very important development of this treatment is the use of interstitial needles as a compliment to the intracavitary applicators for creating a more adapted optimized dose distribution.

The prior intracavitary brachytherapy technique for cervical cancer was often the implantation of applicators, followed directly by treatment with a standard dose distribution without moving the patient $[8,9]$. With the IGABT
Address for correspondence: Leif Karlsson, PhLic, Department of Medical Physics, Örebro University Hospital, SE-70185 Örebro, Sweden, phone: +46196021394, « e-mail: leif.karlsson@regionorebrolan.se
Received: 17.03 .2016

Accepted: 13.02 .2017

Published: 28.02.2017 
technique, the patient is often shifted several times, starting with the transport from the operating theater to the MRI/CT, and finally to the treatment room. This procedure may introduce geometrical uncertainties, since due to these movements, a possible shift of the applicators in relation to the target and OAR may occur between imaging and treatment. The most probable shift of the applicator would be a longitudinal caudal displacement, which would cause a separation between the target and applicator. To avoid a separation, it is crucial to have a solid fixation of the applicator in order to avoid intra-fractional uncertainties. A validation of the treatment geometry can be achieved by comparing the planning geometry (MRI/CT examination) with the existing geometry at dose delivery.

Knowledge of uncertainties in brachytherapy is essential [10,11]. Kirisits et al. [11] proposed guidelines for uncertainties where they identified uncertainty components, such as slice thickness and source positioning error, and their relative importance to the overall uncertainty in brachytherapy. There have also been studies of inter-fraction and intra-fraction uncertainties for both HDR and PDR $[12,13,14,15,16,17,18,19,20,21]$. Intra-fractional geometrical uncertainties due to applicator shift during the time interval (1.5-2 $\mathrm{h}$ ) between imaging and dose delivery in cervix brachytherapy has not been thoroughly investigated $[22,23]$.

At our department, IGABT was introduced in 2007 for the treatment of advanced cervical cancer using a tandem ring applicator. In 2011, this technique was enhanced with the use of interstitial needles and is now utilized in $65 \%$ of patients. This study aims to determine the intra-fractional longitudinal applicator shift between imaging and dose delivery in cervix brachytherapy and its estimated dosimetric impact on the target, HR-CTV for patients with and without needles.

\section{Material and methods}

\section{Patients}

Radiation dose planning data from 71 fractions from 33 patients with cervical cancer who were treated with HDR brachytherapy between January 2012 and May 2015 was retrospectively included in the study, using the following inclusion criteria: 1) gold markers must have been used (for more information, see treatment procedure); 2) dose planning must be based on CT images, since gold markers are difficult to see MRI images [24]. The study was approved by the regional ethics committee (DNR 2015/068).

\section{Treatment procedure}

The radiotherapy treatment consists of external radiotherapy, 46.8-50.4 Gy in 1.8 Gy per fraction, and brachytherapy 4 fractions of 6 or $7 \mathrm{~Gy}$ to a total equivalent dose in $2 \mathrm{~Gy}$ per fraction $\left(\mathrm{EQD}_{2}\right)$ of $\mathrm{D}_{90}>85 \mathrm{~Gy}$. The intracavitary/interstitial HDR brachytherapy was performed as follows: the patient received 2-3 gold markers placed in or adjacent to the cervical tissue before brachytherapy implantation to act as a surrogate for the HR-CTV in the analysis of the applicator shift. The volume of the tissue where the markers should be implanted can be described as a box $4 \times 4 \times 2 \mathrm{~cm}^{3}$ localized approximately $1 \mathrm{~cm}$ cranially to the outer surface of the ring applicator. Gold markers were placed in between the left and right parts of the box.

At the time of implantation, an interstitial ring applicator (Nucletron, an Elekta company, Elekta AB, Stockholm, Sweden) was used. The intracavity ring was positioned in the vaginal vault and the tube located intrauterine. Interstitial needles were added through the ring cap, when deemed necessary, in order to cover the target volume with an adequate dose. After implantation, the applicator was fixed by packing of the vagina. A Foley catheter was placed in the bladder, and pulled towards the bladder base and fixed. The fixation of the Foley catheter also acted as a geometric stabilizer. The patient was transported for MRI/CT examination. In the images obtained from the examination, the high risk clinical target volume (HR-CTV) was defined according to European recommendations from the GEC-ESTRO Working Group (I) [4]. Organs at risk (OAR) such as the bladder, rectum, and sigmoid were also defined. In the same image set, the applicators were reconstructed and an optimized dose distribution based on the dose constraints for the HR-CTV and OAR were created. This procedure was repeated for all four fractions, two fractions per week, and with one fraction delivered per insertion. Fractions 1 and 3 were based on an MRI examination, and fractions 2 and 4 were based on a CT examination. However, for five patients included in this study, three CT examinations had been performed but the images for the first fraction was in all cases acquired with MRI. The CT images were co-registered with the MRI images to visualize the target outlined using the MRI images in the CT images, as described by Nesvacil et al. [25]. The bladder was emptied and refilled to a fixed liquid volume of $50 \mathrm{~cm}^{3}$, and a catheter was inserted into the rectum to prevent any gas filling before both imaging and dose delivery. The procedure described above includes two disturbances of the patient, which could induce applicator shifts, and consequently, geometrical uncertainties. To be able to calculate an estimate of a possible applicator shift between planning CT imaging and dose delivery, a quick and easy measurement method was introduced. Two semi-orthogonal radiographs were obtained, frontal and lateral views just before treatment. In these images, the interstitial ring applicator, X-ray markers, and the implanted gold markers were identified. Possible geometrical shifts of the applicators were then estimated by measurements in both the CT images used for planning and the radiographs obtained just prior to dose delivery.

\section{In vivo study}

On the radiographs, the gold markers and X-ray markers in the ring-applicator can be visualized. The interstitial ring applicator consists of two parts: an IU tube, which was 2-6 cm long, and a ring with a diameter of $26 \mathrm{~mm}$ or $30 \mathrm{~mm}$ (source path diameter). These two parts were perpendicular to each other (Figure 1). A coordinate system was created in the lateral radiograph with the 
A

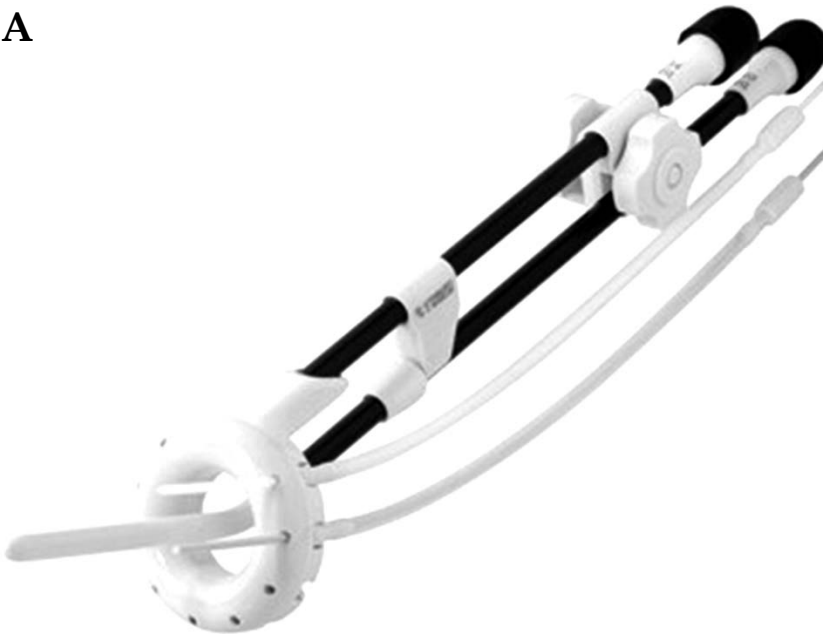

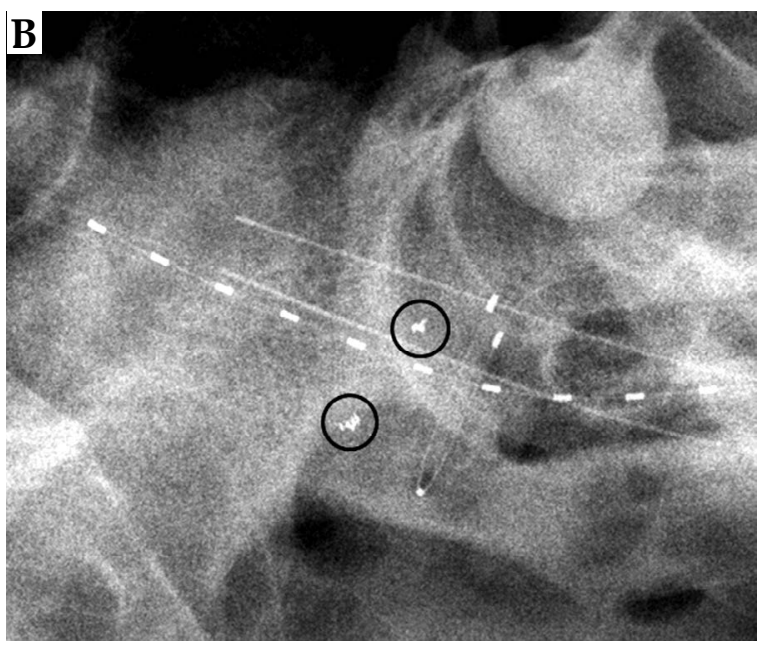

Fig. 1. A) The interstitial ring applicator. B) The interstitial ring applicator placed in vivo visualized in a radiograph by X-ray markers in the ring and in the intrauterine tube. Two gold markers are also visible in the radiograph, marked with black circles

Z-axis, along the IU part and the XY-plane (ring plane) through the source path in the ring, described by the $X$-ray markers. Distance measurements were made on the lateral radiograph where the $\mathrm{X}$-ray markers in the ring applicator and gold markers were projected (Figure 2A). The gold markers were divided between the left and right side of the ring applicator, and a mean distance was calculated from the left side and right side measurements in the radiographs. This was done to minimize the influence of the divergence in the lateral radiograph. For patients with three gold markers, the third marker was nevertheless included in the calculation of the mean val-

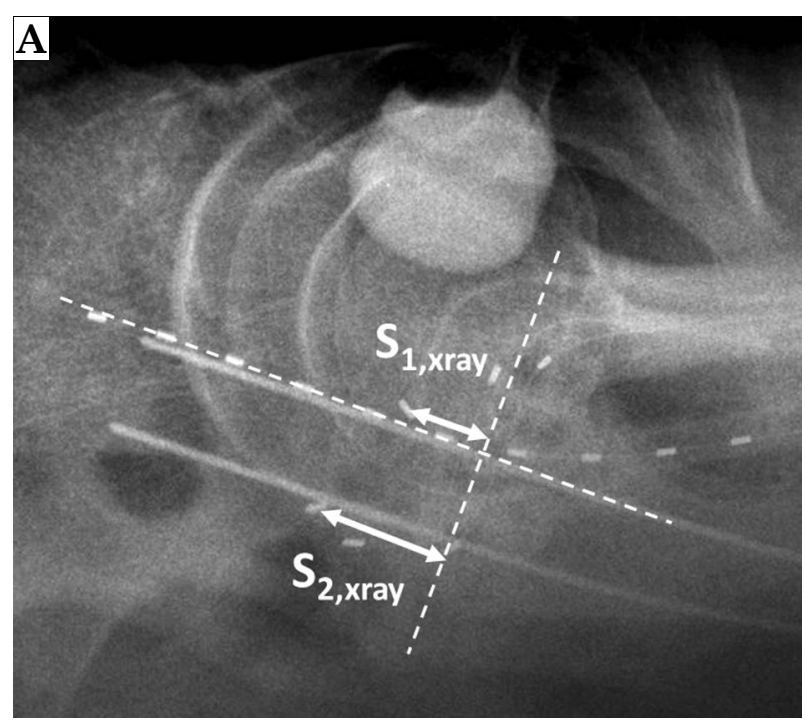

Fig. 2. A) Image just before treatment showing the X-ray markers defining the ring applicator and the coordinate system, with the $\mathrm{Z}$-axis along the intra uterine catheter and the $X Y$-plane in the ring plane. $S_{1, \text { xray }}$ and $S_{2, \text { xray }}$ were two measured distances between the gold markers to the ring plane. B, C) Reconstructed CT images show the corresponding measurements, $\mathrm{S}_{1, \mathrm{CT}}$, and $\mathrm{S}_{2, \mathrm{CT}}$ at the time of imaging ue, because the effect of divergence was shown (in the uncertainty analysis below) to be minimal.

The CT examination contains images with a slice thickness of $2 \mathrm{~mm}$, orientated parallel with the ring plane. Digitally reconstructed planes with the gold marker were prepared in the CT images (Figures 2B, 2C). All CT measurements were performed in the treatment planning system (TPS), Oncentra Brachy (OCB), (Nucletron, an Elekta company, Elekta AB, Stockholm, Sweden).

Measurements of the longitudinal shift were performed in all 71 fractions, 25 without needles, and 46 with needles. The individual fractions from a patient were as-
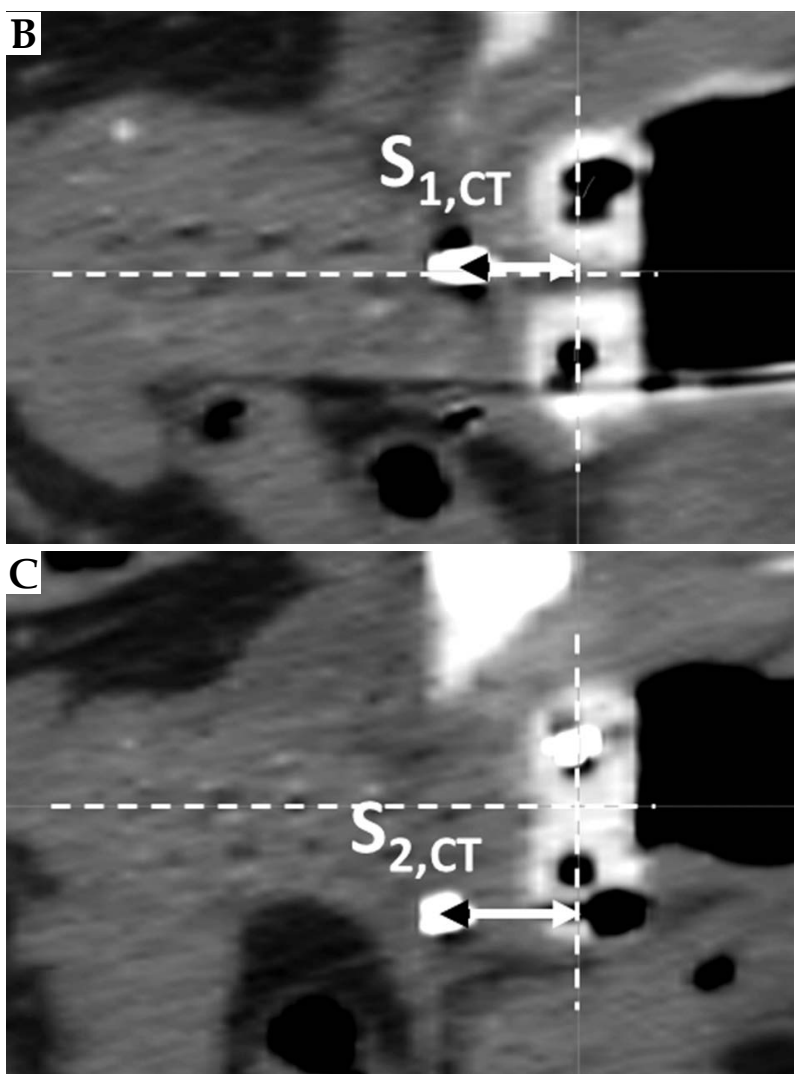
sumed to be geometrically independent of each other in the analysis of applicator shift.

\section{Uncertainty analysis of the in vivo measurement method}

An estimation of the uncertainty in the measurement method was made as a validation for the measurements in vivo. The source of uncertainties from the radiograph was assumed to arise from the divergence, rotation of the applicator, and the gold markers in the XZ-plane and the magnification factor. Rotations greater than 3 degrees were corrected during the in vivo measurements in the $\mathrm{CT}$ images. An in-house phantom was created. This consisted of a ring applicator and eight fiducial markers placed at predefined positions (Figure 3). The phantom was depicted with the $\mathrm{CT}$ and with radiographs in the treatment room. To simulate different patient positions in the treatment room, the phantom was positioned in 10 different positions, no rotation, rotated \pm 5 , and \pm 10 degrees in the XZ-plane with no rotation in the $\mathrm{XY}$-plane, and this was repeated with a 5-degree rotation in the $\mathrm{XY}$-plane to simulate possible rotations of the applicator in the coordinate system. The gold markers' mean values were calculated from the measured distance for the left marker and the corresponding marker on the right side of the ring applicator, to consider the divergence in the radiographs and the rotation of the applicator. Since the configuration of the phantom was identical in both the radiographs and CT images, there should ideally be a difference of zero between the measurements.

In the clinical situation, the gold markers were placed by hand on the left and right side of the intrauterine applicator into the tissue box, so the ideal situation, which applies to the phantom described above, doesn't exist and the mean value calculation will not fully correct for the divergence. The discrepancy was therefore determined for a gold marker placed anywhere inside the tissue box, and this was found to be $\pm 0.2 \mathrm{~mm}$.

A possible migration of the gold markers was also analyzed for a small sample (17) of the fractions by observing the stability of the markers relative to each other on the $\mathrm{CT}$ and on the lateral radiographs.

\section{Analysis of the dosimetric impact on HR-CTV due to applicator shift}

To investigate the influence of the applicator shift on the dose coverage of the HR-CTV, the dose volume parameter $D_{90}$ was used, which was defined as the dose to the $90 \%$ of the HR-CTV volume. In the TPS, the original dose distribution $\left(\mathrm{D}_{90 \mathrm{ref}}\right)$ was shifted according to the individually measured applicator displacement to give a new dose distribution $\left(\mathrm{D}_{90 \mathrm{i}}\right)[26,27]$. To access the dosimetric changes in $\mathrm{D}_{90} \mathrm{HR}-\mathrm{CTV}$ due to applicator shift, the relative differences in physical dose of $\mathrm{D}_{90} \mathrm{HR}-\mathrm{CTV}$ for each fraction was then calculated as $\Delta D=\left(D_{i}-D_{\text {ref }}\right) /$ $\mathrm{D}_{\text {ref }}(\%)$ as recommended by Nesvacil et al. [21].

\section{Statistics}

Data was checked for normality using the Shapiro-Wilks test. All groups were then checked for significance $(p<0.05)$ using the paired or one sample students $t$-test if the data had a normal distribution (parametrical test), and the Wilcoxon Signed Ranks Test (non-parametrical test) for data without normal distribution. When comparing the two
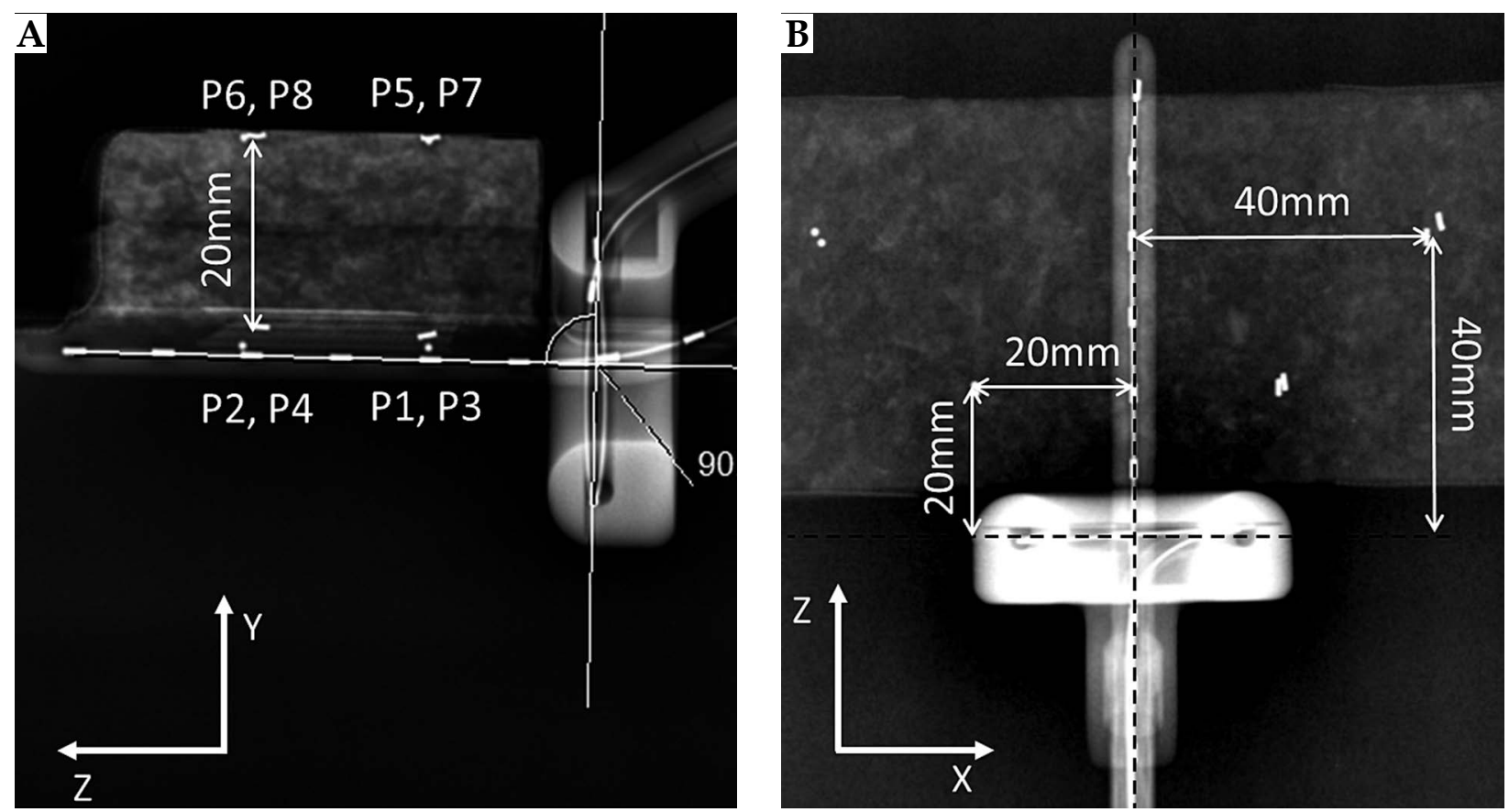

Fig. 3. Radiographs showing the phantom used for the in vitro measurements. The phantom consists of a ring applicator and eight markers (P1-P8) placed in a predefined way. A) A lateral view with the markers shown and the distance between the two marker planes. B) A frontal view with the distances from the markers to the coordinate system. The Z-axis is along the intra-uterine tube and the XY-plane in the ring plane 
Table 1. Applicator shift from the in vivo study. A negative sign means a cranial displacement while a positive sign implies a caudal displacement

\begin{tabular}{|c|c|c|c|c|c|}
\hline Applicator shift (mm) & $n$ & Mean/Median & $\mathrm{SD}$ & $95 \% \mathrm{Cl}$ & Range \\
\hline All fractions & 71 & $-0.2 /-0.4$ & 1.1 & $-0.51,0.02$ & $-2.5,3.6$ \\
\hline Fractions without needles & 25 & $-0.7 /-0.6$ & 0.9 & $-1.03,-0.28$ & $-2.5,0.7$ \\
\hline Fractions with needles & 46 & $0.0 /-0.1$ & 1.2 & $-0.37,0.32$ & $-2.1,3.6$ \\
\hline
\end{tabular}

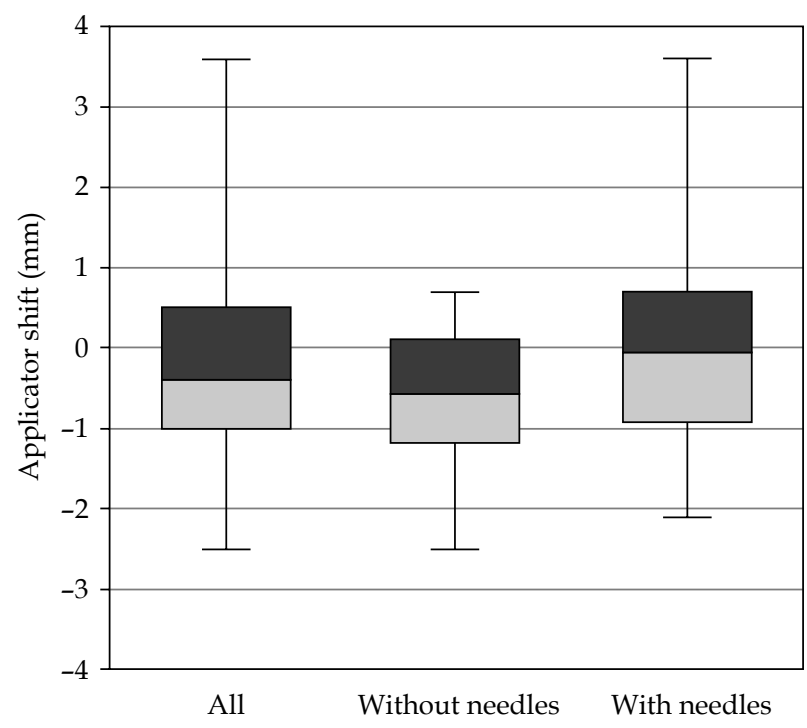

Fig. 4. Box plot showing a comparison between the distributions of the determined applicator shifts for all (71 fractions) without needles (25 fractions) and with needles (46 fractions). A negative sign means a cranial applicator displacement and a positive sign a caudal displacement

groups with and without needles, unpaired students $t$-test (parametrical data) and Mann-Whitney $U$ test (non-parametrical data) was used. The analytical statistics was presented as $95 \%$ confidence interval (CI) or $p$-value. The SPSS software was used for the statistical analysis.

\section{Results}

\section{In vivo study}

The results from the displacement measurements are presented in Table 1 and Figure 4. The maximum and minimum applicator shift was $3.6 \mathrm{~mm}$ and $-2.5 \mathrm{~mm}$, respectively. A negative sign implies a cranial shift and a positive sign as a caudal shift. For the group without needles, there was a significant cranial displacement of the applicator shift between imaging and dose delivery, although for the group with needles, there was no significant shift. There was a significant difference in displacement between the group with needles and the group without needles $(p=0.022)$.

\section{Uncertainty analysis of the in vivo measurement method}

The determined uncertainty from experimental analysis was $0.1 \mathrm{~mm}(\mathrm{SD}=0.4 \mathrm{~mm})$, and confidence interval (95\% CI: -0.05-0.23). The maximum and minimum differences were $1.0 \mathrm{~mm}$ and $-1.0 \mathrm{~mm}$, respectively. The measurement of the migration of the gold markers resulted in a mean variation between the $\mathrm{CT}$ and the radiographs of $1.1 \mathrm{~mm}(\mathrm{SD}=1 \mathrm{~mm})$.

\section{Analysis of the dosimetric impact on HR-CTV}

There was no significant relative dose reduction, $\Delta D_{90}$, for the whole group (Table 2), but there was a dose reduction of up to $6.6 \%$ in individual fractions. There was a significant difference between the groups with and without needles $(p=0.008)$.

\section{Discussion}

In HDR brachytherapy of cervical cancer, it is vital that the dose planning geometry agrees with the treatment geometry. Ideally, there should be no difference between the planning geometry and the treatment geometry. The result of the phantom study shows that there was an insignificant difference between the two geometries when measuring in the radiographs and the CT images. The analysis of the movement of the markers also showed that there was a mean migration of $1.1 \mathrm{~mm}(\mathrm{SD}=1 \mathrm{~mm})$ of the markers relative to each other, and this will add to the overall uncertainty in the measurement method. The direction of the movement is difficult to define. It could

Table 2. Relative differences in physical dose of $D_{90}$ HR-CTV for each fraction. For assessment of the dosimetric changes in $D_{90} H R-C T V$ due to applicator shift between imaging $\left(D_{90 \text { ref }}\right)$ and dose delivery $\left(D_{90 i}\right)$, the relative differences of physical dose was calculated as $\Delta D_{90}=\left(D_{90 i}-D_{90 \text { ref }}\right) / D_{90 \text { ref }}(\%)$. A negative sign means there was a dose reduction in $D_{90}$ HR-CTV at dose delivery

\begin{tabular}{|c|c|c|c|c|c|}
\hline$\Delta \mathrm{D}_{90} \mathrm{HR}-\mathrm{CTV}(\%)$ & $n$ & Mean & SD & $95 \% \mathrm{Cl}$ & Range \\
\hline All fractions & 71 & 0.6 & 2.4 & $-0.01,1.13$ & $-6.6,6.8$ \\
\hline Fractions without needles & 25 & 1.6 & 2.6 & $0.53,2.64$ & $-2.9,6.8$ \\
\hline Fractions with needles & 46 & 0.0 & 2.2 & $-0.64,0.66$ & $-6.6,6.6$ \\
\hline
\end{tabular}


be in any direction, also a radial movement relative the IU applicator and in that case, it does not affect the measurement of the applicator shift. Using the combined standard uncertainty of these two uncertainties $( \pm 2.1 \mathrm{~mm}, \mathrm{k}=2)$ as an estimate of the overall uncertainty, it seems reasonable to accept the measurement method in the in vivo measurements to determine an applicator shift.

The results of this study show a mean physical dose variation due to the applicator shift for $\Delta \mathrm{D}_{90} \mathrm{HR}-\mathrm{CTV}$ of $0.6 \%(\mathrm{SD}=2.4 \%)$. In a previous study, Lang et al. [16] compared two HDR fractions within one insertion, separated in mean by approximately 16 hours, and with transfer of the patient from an MRI-table to the bed. They superimposed the dose distribution from the first fraction on the MRI images for the second fraction and compared it with a new optimized dose distribution for the second fraction. They found somewhat larger variations, especially for the random uncertainties, in $\mathrm{D}_{90}$ for HR-CTV, $-3.0 \%(\mathrm{SD}=11.5 \%$ ).

In a multicenter study, Nesvacil et al. [21] investigated the dosimetric impact on intra-fractional and inter-fractional anatomical variations in cervix cancer brachytherapy. A treatment plan from first fraction was transferred to following fractions, and DVH-parameters were calculated. For an intra-application, in an inter-fraction case they found variations in $\Delta \mathrm{D}_{90}$ HR-CTV of $-2.5 \%$ (SD = $10.8 \%)$. These two studies also include other uncertainties than those in the present study, such as re-reconstruction of applicators and re-contouring. Nomden et al. [20] made a similar to our study investigation of intra-fraction uncertainties between imaging and dose delivery. They did not however, measure the applicator shift. They compared $\mathrm{D}_{90}$ of the HR-CTV on the planning MRI and the HR-CTV from the pre-treatment MRI (separated by in mean by 3.9 hours), resampled on the planning MRI. They found a difference in the $\mathrm{D}_{90}$ of $-0.1 \mathrm{~Gy}(\mathrm{SD}=0.5 \mathrm{~Gy}), \mathrm{EQD}_{2}$ (equivalent dose at $2 \mathrm{~Gy}$ ). Recalculating the results from this study for comparison gives $0.1 \mathrm{~Gy}(\mathrm{SD}=0.4 \mathrm{~Gy}) \mathrm{EQD}_{2}$.

Even if the random uncertainties were low for the relative differences of $\mathrm{D}_{90} \mathrm{HR}-\mathrm{CTV}$, there were some extreme values of dose reduction due to the measured applicator shifts. The maximum dose reduction was $6.6 \%$. Therefore, a dosimetric effect of $6.6 \%$ will result in a reduction of around $5 \mathrm{~Gy} \mathrm{EQD}$, which is not clinically negligible if the same shift was present for all 4 fractions.

A possible limitation of this study was that only fractions based on CT images were used in the analysis. The fractions with CT images were used due to the difficulties in finding the gold markers in the MRI images. When comparing the logistics between the CT and MRI examinations, there were almost no differences owing to the fact that the patient needed to be lifted and transported in the same manner. The highest risk of introducing geometrical shifts is when moving and lifting the patient, not during transportation of the patient. It was therefore reasonable to assume that the results would be the same for fractions using MRI-data.

Using gold markers as a surrogate for HR-CTV could also be a limitation. There were some unexpected applicator displacements in the cranial direction, which may seem remarkable. This cranial displacement could, to some extent, be explained by the uncertainties in the measurement method, but the cranial displacement measured for ring applicator without needles was significant, and there was a significant difference between the groups with and without using needles. Possible explanations could be a general swelling of the cervix and the adjacent tissue during the insertion of the applicator and a risk of edema/hematoma when using interstitial needles. The results from the analysis of the applicator shift shows a small but significant shift in the cranial direction when using the ring applicator without needles, but looking at the random uncertainties (1SD) of $1.1 \mathrm{~mm}, 1.2 \mathrm{~mm}$, and $0.9 \mathrm{~mm}$ for all, the sub groups with and without needles, respectively, the results are almost the same. Comparing with the estimated overall uncertainty in the measurement method, it is probably difficult to distinguish the difference between using and not using needles.

A few of the gold markers were, during the data collection, found to be in the rectum, sigmoid, or in the bladder wall. These were excluded from the analysis. A displacement of a marker independently of the target, could also occur if for example the bladder filling was changed or the rectum was filled with gas. To avoid these potential problems, a bladder filling of $50 \mathrm{cc}$ was maintained during both imaging and treatment. A catheter in the rectum was used to evacuate any buildup of gas, as previously mentioned.

In a recent review [11], an overall uncertainty of $5 \%$ in $\mathrm{D}_{90}$ was found for HR-CTV under optimal conditions. Intra-fractional geometrical uncertainties due to applicator shifts in close relation (gold markers) to HR-CTV have, to the best of our knowledge, not previously been investigated, and the findings in this study will contribute to the science in this area of overall uncertainties. Applicator shifts have been investigated with bone structures as a reference $[22,23]$, but large movements of the target applicator volume in relation to the bone structures can occur.

This study shows that there were no major changes in the interstitial ring applicator HR-CTV geometry for most of the fractions, between the imaging for planning and at the time of treatment, including a long transportation and several movements of the patient. The main reason for this is probably a rigid fixation of the applicators. The small displacement will not influence the treatment for the majority part of patients, but for some it may be necessary to make an adjustment of the dose distribution. For that reason, there should be a way to detect and quantify any displacements, which might occur between imaging and the time of treatment. The measurement method described here will be able to detect an applicator shift of more than $3 \mathrm{~mm}$. It is a quick method (completed in a matter of minutes) and easily performed while the patient is in the treatment position, but with the restriction of only allowing examination of the cervix. To be able to monitor the whole anatomy such as the bladder and rectum, there would need to be a possibility of using MRI, $\mathrm{CT}$, or CBCT in the treatment position.

\section{Conclusions}

The results from this study showed that there was a small longitudinal displacement of the ring applicator and a significant difference in displacement, between using interstitial needles and not. The mean displacement 
had a minor impact on $\mathrm{D}_{90} \mathrm{HR}-\mathrm{CTV}$, which can be considered as negligible for most patients, but for some patients it can result in an under dosage of the HR-CTV, and therefore it is important to determine the intra-fractional variations between planning and dose delivery. With a good fixation, it is possible to successfully transport and move a patient for MRI/CT imaging without significantly impacting the results of covering HR-CTV.

\section{Disclosure}

Authors report no conflict of interest.

\section{References}

1. Lanciano RM, Won M, Coia LR et al. Pretreatment and treatment factors associated with improved outcome in squamous cell carcinoma of the uterine cervix: a final report of the 1973 and 1978 patterns of care studies. Int J Radiat Oncol Biol Phys 1991; 20: 667-676.

2. Mabuchi S, Okazawa M, Isohashi F et al. Radical hysterectomy with adjuvant radiotherapy versus definitive radiotherapy alone for FIGO stage IIB cervical cancer. Gynecol Oncol 2011; 123: 241-247.

3. Chai Y, Wang T, Wang J et al. Radical hysterectomy with adjuvant radiotherapy versus radical radiotherapy for FIGO stage IIB cervical cancer. BMC Cancer 2014; 14: 63.

4. Haie-Meder C, Pötter R, Van Limbergen E et al. Recommendations from Gynaecological (GYN) GEC-ESTRO Working Group (I): concepts and terms in 3D image based 3D treatment planning in cervix cancer brachytherapy with emphasis on MRI assessment of GTV and CTV. Radiother Oncol 2005; 74: 235-245.

5. Pötter R, Haie-Meder C, Van Limbergen E et al. Recommendations from gynaecological (GYN) GEC ESTRO Working Group (II): concepts and terms in 3D image-based treatment planning in cervix cancer brachytherapy-3D dose volume parameters and aspects of 3D image-based anatomy, radiation physics, radiobiology. Radiother Oncol 2006; 78: 67-77.

6. Dimopoulos JC, Petrow P, Tanderup K et al. Recommendations from Gynaecological (GYN) GEC-ESTRO Working Group (IV): Basic principles and parameters for MR imaging within the frame of image based adaptive cervix cancer brachytherapy. Radiother Oncol 2012; 103: 113-122.

7. Hellebust TP, Kirisits C, Berger D et al. Recommendations from Gynaecological (GYN) GEC-ESTRO Working Group: considerations and pitfalls in commissioning and applicator reconstruction in 3D image-based treatment planning of cervix cancer brachytherapy. Radiother Oncol 2010; 96: 153-160.

8. Sorbe B, Bohr L, Karlsson L et al. Combined external and intracavitary irradiation in treatment of advanced cervical carcinomas: predictive factors for local tumor control and early recurrences. Int J Oncol 2010; 36: 371-378.

9. Bohr Mordhorst L, Karlsson L, Bärmark B et al. Combined external and intracavitary irradiation in treatment of advanced cervical carcinomas: predictive factors for treatment outcome and early and late radiation reactions. Int J Gynecol Cancer 2014; 24: 1268-1275.

10. Palmer A, Bradley D, Nisbet A. Physics-aspects of dose accuracy in high dose rate (HDR) brachytherapy: source dosimetry, treatment planning, equipment performance and in vivo verification techniques. J Contemp Brachytherapy 2012; 4: 81-91.

11. Kirisits C, Rivard MJ, Baltas D et al. Review of clinical brachytherapy uncertainties: analysis guidelines of GEC-ESTRO and the AAPM. Radiother Oncol 2014; 110: 199-212.
12. Hellebust TP, Dale E, Skjonsberg A et al. Inter fraction variations in rectum nd bladder volumes and dose distributions during high dose rate brachytherapy treatment of the uterine cervix investigated by repetitive CT-examinations. Radiother Oncol 2001; 60: 273-280.

13. Kirisits C, Lang S, Dimopoulos J et al. Uncertainties when using only one MRI-based treatment plan for subsequent highdose-rate tandem and ring applications in brachytherapy of cervix cancer. Radiother Oncol 2006; 81: 269-275.

14. Tanderup K, Christensen JJ, Granfeldt J et al. Geometric stability of intracavitary pulsed dose rate brachytherapy monitored by in vivo rectal dosimetry. Radiother Oncol 2006; 79: 87-93.

15. Beriwal S, Kim H, Coon D et al. Single magnetic resonance imaging vs. magnetic resonance imaging/computed tomography planning in cervical cancer brachytherapy. Clin Oncol (R Coll Radiol) 2009; 21: 483-487.

16. Lang S, Nesvacil N, Kirisits C et al. Uncertainty analysis for 3D image-based cervix cancer brachytherapy by repetitive MR imaging: assessment of DVH-variations between two HDR fractions within one applicator insertion and their clinical relevance. Radiother Oncol 2013; 107: 26-31.

17. Morgia M, Cuartero J, Walsh L et al. Tumor and normal tissue dosimetry changes during MR-guided pulsed-dose-rate (PDR) brachytherapy for cervical cancer. Radiother Oncol 2013; 107: 46-51.

18. Anderson C, Lowe G, Wills R et al. Critical structure movement in cervix brachytherapy. Radiother Oncol 2013; 107: 39-45.

19. Chakraborty S, Patel FD, Patil VM et al. Magnitude and Implications of Interfraction Variations in Organ Doses during High Dose Rate Brachytherapy of Cervix Cancer: A CT Based Planning Study. ISRN Oncol 2014; 2014: 687365.

20. Nomden CN, de Leeuw AA, Roesink JM et al. Intra-fraction uncertainties of MRI guided brachytherapy in patients with cervical cancer. Radiother Oncol 2014; 112: 217-220.

21. Nesvacil N, Tanderup K, Hellebust TP et al. A multicentre comparison of the dosimetric impact of inter- and intra-fractional anatomical variations in fractionated cervix cancer brachytherapy. Radiother Oncol 2013; 107: 20-25.

22. De Leeuw AA, Moerland MA, Nomden C et al. Applicator reconstruction and applicator shifts in 3D MR-based PDR brachytherapy of cervical cancer. Radiother Oncol 2009; 93: 341-346.

23. Shi D, He MY, Zhao ZP et al. Utrecht Interstitial Applicator Shifts and DVH Parameter Changes in 3D CT-based HDR Brachytherapy of Cervical Cancer. Asian Pac J Cancer Prev 2015; 16: 3945-3949.

24. Soliman AS, Owrangi A, Ravi A et al. Metal artefacts in MRI-guided brachytherapy of cervical cancer. J Contemp Brachytherapy 2016; 8: 363-369.

25. Nesvacil N, Potter R, Sturdza A et al. Adaptive image guided brachytherapy for cervical cancer: a combined MRI-/CT-planning technique with MRI only at first fraction. Radiother Oncol 2013; 107: 75-81.

26. Schindel J, Zhang W, Bhatia SK et al. Dosimetric impacts of applicator displacements and applicator reconstruction-uncertainties on 3D image-guided brachytherapy for cervical cancer. J Contemp Brachytherapy 2013; 5: 250-257.

27. Karlsson L, Thunberg P, Johansson B et al. The impact of activating source dwell positions outside the CTV on the dose to treated normal tissue volumes in TRUS guided 3D conformal interstitial HDR brachytherapy of prostate cancer. J Contemp Brachytherapy 2014; 6: 282-288. 